\title{
Lymphocyte-derived microparticles stimulate osteoclastogenesis by inducing RANKL in fibroblasts of odontogenic keratocysts
}

\author{
QI-WEN MAN ${ }^{1 *}$, LIN-ZHOU ZHANG $^{1 *}$, YI ZHAO $^{2}$, JIN-YUAN LIU $^{1}$, YUE-YU ZHENG ${ }^{1}$, \\ YI-FANG ZHAO ${ }^{1,3}$ and BING LIU ${ }^{1,3}$ \\ ${ }^{1}$ The State Key Laboratory Breeding Base of Basic Science of Stomatology and \\ Key Laboratory of Oral Biomedicine Ministry of Education, Wuhan University School of Stomatology; \\ ${ }^{2}$ Department of Prosthodontics, School and Hospital of Stomatology; ${ }^{3}$ Department of Oral and Maxillofacial Surgery, \\ Wuhan University School of Stomatology, Wuhan University, Wuhan, Hubei 430049, P.R. China
}

Received February 18, 2018; Accepted September 3, 2018

DOI: $10.3892 / o r .2018 .6708$

\begin{abstract}
Leukocyte-derived microparticles (LMPs) include neutrophil-, lymphocyte- and monocyte-derived MPs. LMPs act as proinflammatory mediators in autoimmune diseases, infectious diseases and vascular diseases. The present study examined the hypothesis that the percentage of LMPs was increased in patients with inflamed odontogenic keratocysts (OKCs), and investigated the biological effects of Jurkat cell-derived MPs on the fibroblasts of OKCs in vitro. Cyst fluid MPs, obtained by centrifugation of samples from 20 patients with inflamed OKCs, 3 patients with uninflamed OKCs, 15 patients with radicular cysts (RCs) and 12 patients with inflamed dentigerous cysts (DCs), were analyzed by transmission electron microscopy, dynamic light scattering and immunofluorescence staining. The percentages and concentrations of cyst fluid LMPs were further determined by flow cytometry. The cytokine levels of apoptotic Jurkat cell-derived MPs and Jurkat cell supernatants were compared by cytokine antibody arrays. Fibroblasts were isolated from 3 patients with OKC and co-cultured with apoptotic Jurkat cell-derived MPs with or without interleukin (IL)-15R $\alpha$ to detect the levels of matrix metallopeptidase 9 (MMP-9) and receptor activator of nuclear factor- $\kappa \mathrm{B}$ ligand (RANKL) by reverse transcription-quantitative polymerase chain reaction and enzyme-linked immunosorbent assay. The supernatant from Jurkat MPs-treated fibroblasts was collected to make conditioned medium in which the osteoclastogenesis of
\end{abstract}

Correspondence to: Professor Bing Liu, Department of Oral and Maxillofacial Surgery, Wuhan University School of Stomatology, Wuhan University, 237 Luoyu Road, Wuhan, Hubei 430049, P.R. China

E-mail: liubing9909@whu.edu.cn

*Contributed equally

Key words: cyst fluid, odontogenic keratocysts, leukocyte-derived microparticles, inflammation
Raw264.7 cells was determined. Antibodies against human soluble (s)RANKL were added to the conditioned medium to investigate the inhibitory effects. Mean percentages of lymphocyte- and neutrophil-derived MPs were significantly higher in inflamed OKCs than in DCs. Significant elevations in IL-15 were detected in apoptotic Jurkat cell-derived MPs compared with that in Jurkat cell supernatant. Furthermore, higher levels of MMP-9 and RANKL were detected in Jurkat cell MP-treated OKC fibroblasts, and this was partially blocked by IL-15R $\alpha$. Increased osteoclast-like cell formation was observed in the Jurkat MPs-treated fibroblast supernatant and Raw264.7 co-culture groups. The anti-human sRANKL antibody in the Jurkat MPs-treated fibroblast supernatant group decreased the osteoclastogenesis of the Raw264.7 cells. These results indicate that LMPs serve as novel communication tools that contribute toward the bone resorption of inflamed OKCs by inducing RANKL of OKC fibroblasts via IL-15.

\section{Introduction}

Odontogenic keratocysts (OKCs), previously defined as keratocystic odontogenic tumors, are common jaw cysts with the potential to be aggressive and the tendency to recur (1). An extensive volume of research has demonstrated that the epithelium of OKCs has a unique growth potential compared with that of other cysts $(2,3)$. In particular, there is ample evidence that a large number of OKCs (sporadic and syndromic) harbor patched 1 gene mutations, and the aggressive growth potential of these developmental cysts have been attributed to this $(2,3)$. Although OKC belongs to a group of developmental jaw cysts (4), it is frequently exposed to microorganisms and the majority of OKCs often occur along with focal inflammatory infiltrates (5). Radicular cysts (RCs) are the most common jaw cysts, accounting for $52 \%$ of jaw cystic lesions (6). RCs are a result of the inflammatory process in the periapical tissues associated with necrotic and infected pulps, and usually occur along with diffused inflammatory cell infiltrations (6). It is widely accepted that the cytokines from neutrophils, monocytes and other immune system cells could facilitate the growth and enlargement of RC (7). Dentigerous cysts (DCs) are associated with the crown of an unerupted tooth, with inflammation 
ranging between little to relatively extensive (8). Cysts of the jaw lesions are known as common osseous-destructive lesions. The inflammatory cells, upon stimulation, are able to release cytokines and other undetected functional protein carriers into the cyst cavity. It is possible that variations in the inflammatory infiltrate could result in the different osteolytic activity.

Cell-derived microparticles (MPs) are small (100-1,000 nm in diameter) membrane-enclosed vesicles secreted from cells by direct budding from the plasma membrane (9). Nearly all eukaryocytes can release MPs. Among them, leukocyte-derived MPs (LMPs) originate from neutrophils, monocytes/macrophages and lymphocytes (10). LMPs express markers from their parental cells, including cluster of differentiation (CD)4, CD14 and CD15. Given that they arise from the rearrangement of the plasma, MPs also present with phosphatidylserine (PS) on their surface. Therefore, a previous study used the mother cell antigens and Annexin V detection in flow cytometry to positively define MPs (11). The invasion of bacteria or bacterial toxins into the jaw cystic cavity involves initial inflammatory reactions and leads to the accumulation of leukocytes $(5,12)$. Since odontogenic cysts are directly immersed in the milieu of cyst fluids, leukocytes may release MPs into the cavity. However, whether LMPs are present in the cyst fluid of odontogenic lesions and their biological functions remain unknown.

To the best of our knowledge, the present study was the first to demonstrate increased percentages of LMPs in inflamed OKCs compared with those in DCs. It was also demonstrated that MPs derived from leukocytes express IL-15 and other cytokines that potently induce the expression of RANKL and MMP-9 in OKC fibroblasts, which promotes osteoclastogenesis. The present study revealed a novel mechanism for the lymphocyte-induced bone resorption of OKCs.

\section{Materials and methods}

Study population. All procedures were performed according to the National Institutes of Health guidelines regarding the use of human tissues, and the present study was approved by the Review Board of the Medical Ethics Committee of the Hospital of Stomatology, Wuhan University (Wuhan, Hubei, China) and was conducted between April 2016 and August 2017. All patients provided written informed consent to participate in the study and for its publication. The present study included a total of 20 patients with focal inflamed OKC, 3 patients with uninflamed $\mathrm{OKC}, 15$ patients with $\mathrm{RC}$ and 12 patients with focal inflamed DCs, diagnosed according to the fourth edition of the World Health Organization Classification of Head and Neck Tumors (13). Since diffused inflamed or severe inflamed OKCs or DCs are difficult to diagnose, the present study only included focal inflamed OKCs or DCs. Patients with nevoid basal cell carcinoma syndrome-related OKCs were excluded from the study. Hematoxylin and eosin (HE) staining of odontogenic cysts was performed for diagnostic purposes.

Cyst fluid collection and isolation of cyst fluid MPs. Samples of cystic fluids were obtained from the cyst cavities by aspiration using a syringe attached to an $18 \mathrm{G}$ sterilized needle prior to marsupialization or enucleation. Cyst fluids were immediately centrifuged at $3,000 \times \mathrm{g}$ for $20 \mathrm{~min}$ at $4^{\circ} \mathrm{C}$ (centrifuge 5810R;
Eppendorf, Hamburg, Germany). Supernatants were collected and centrifuged at $3,000 \mathrm{x}$ g for another $20 \mathrm{~min}$ to obtain cell-free cyst fluids. Subsequently, the supernatants were diluted with an equal volume of $\mathrm{Ca}^{2+} / \mathrm{Mg}^{2+}$-free highly purified phosphate-buffered saline (PBS; Beyotime Institute of Biotechnology, Haimen, China) and were then centrifuged at $10,000 \mathrm{x} \mathrm{g}$ for $40 \mathrm{~min}$ at $4^{\circ} \mathrm{C}$. This step aimed to remove apoptotic bodies and larger vesicles. Next, the supernatants were centrifuged at 50,000 $\mathrm{x}$ g for $1 \mathrm{~h}$ at $4^{\circ} \mathrm{C}$ (Avanti J-26 XP; Beckman Coulter, Inc., Brea, CA, USA) to pellet cystic fluid MPs (CFMPs) as previously described (14). CFMP pellets were resuspended in $150 \mu \mathrm{l}$ PBS. Subsequently, $50 \mu \mathrm{l}$ of each CFMP sample was prepared for flow cytometry and the other $100 \mu \mathrm{l}$ was immediately stored at $-80^{\circ} \mathrm{C}$ for further study.

Characterization of CFMPs by transmission electron microscopy (TEM). TEM was performed at the Wuhan Institute of Virology (Wuhan, China). Freshly-isolated CFMPs and Jurkat cell-derived MPs were placed on a copper grid for observation. The grids were stained with $1 \% \mathrm{v} / \mathrm{v}$ uranyl acetate and the samples were examined on a Hitachi HT7700 transmission electron microscope (Hitachi, Ltd., Tokyo, Japan).

Characterization of CFMPs by carboxyfluorescein succinimidyl ester (CFSE) staining. The freshly isolated CFMPs were incubated with $10 \mu \mathrm{M}$ CFSE (Thermo Fisher Scientific, Inc., Waltham, MA, USA) at $37^{\circ} \mathrm{C}$ for $30 \mathrm{~min}$ in the dark. Subsequently, the samples were analyzed using a fluorescence microscope (Leica Microsystems GmbH, Wetzlar, Germany) or a FACSAria II flow cytometer (BD Biosciences, Franklin Lakes, NJ, USA).

Characterization of CFMPs by dynamic light scattering. The hydrodynamic diameters of CFMPs were determined by a Nano-ZS ZEN 3600 (Malvern Instruments, Inc., Westborough, MA, USA), which was equipped with a He-Ne laser (633 nm), as previously described (15).

Detection and analysis of CFMPs by flow cytometry. Flow cytometric analysis was performed using a BD LSRFortessa flow cytometer (BD Biosciences). The size of CFMPs was identified using Nile Red particles with a diameter of 0.7-0.9 $\mu \mathrm{m}$ (Spherotech, Lake Forest, IL, USA). To quantify the CFMPs, a known number of calibrator flow-count fluorosphere beads (10- $\mu \mathrm{m}$ diameter; Beckman Coulter, Inc.) was added to determine the number of CFMPs. As flow-count fluorospheres have a definite concentration, when identical volumes of a sample and flow-count fluorospheres were added and tested, the concentration of CFMPs could be calculated using the following formula: CFMPs concentration $=$ (total number of events for the sample/total number of events for flow-count fluorospheres) $\mathrm{x}$ flow-count fluorosphere assayed concentration. Next, the concentrations of CFMPs were calculated using the initial volumes of cyst fluids listed in Tables I-IV. The subpopulations of CFMPs were detected according to membrane-specific antigens. Subsets of the CFMPs were identified as follows: Monocyte-derived MPs $\left(\mathrm{CD}_{14}{ }^{+} /\right.$Annexin $\left.\mathrm{V}^{+}\right)$, neutrophil-derived MPs (CD15+/Annexin $\mathrm{V}^{+}$) and lymphocyte-derived MPs $\left(\mathrm{CD}^{+} /\right.$Annexin $\mathrm{V}^{+}$). Antibodies were added to $10 \mu \mathrm{l}$ CFMPs 
Table I. Summary of clinical features of patients with focal inflamed dentigerous cysts.

\begin{tabular}{|c|c|c|c|c|}
\hline Patient no. & Sex & Age, years & Location & $\begin{array}{l}\text { Cyst fluid } \\
\text { drained, ml }\end{array}$ \\
\hline 1 & $\mathrm{~F}$ & 30 & Left maxilla & 5 \\
\hline 2 & $\mathrm{~F}$ & 18 & Right mandible & 2 \\
\hline 3 & $\mathrm{M}$ & 18 & Maxilla & 3 \\
\hline 4 & $\mathrm{~F}$ & 33 & Right maxilla & 2 \\
\hline 5 & M & 51 & Right mandible & 9 \\
\hline 6 & M & 42 & Left maxilla & 2 \\
\hline 7 & $\mathrm{~F}$ & 50 & Left mandible & 11 \\
\hline 8 & M & 34 & Right mandible & 3.5 \\
\hline 9 & M & 34 & Right mandible & 2 \\
\hline 10 & M & 56 & Right mandible & 4 \\
\hline 11 & $\mathrm{~F}$ & 28 & Left maxilla & 5 \\
\hline 12 & M & 36 & Right maxilla & 4 \\
\hline
\end{tabular}

M, male; F, female.

Table II. Summary of clinical features of patients with radicular cysts.

\begin{tabular}{|c|c|c|c|c|}
\hline Patient no. & Sex & Age, years & Location & $\begin{array}{l}\text { Cyst fluid } \\
\text { drained, ml }\end{array}$ \\
\hline 1 & $\mathrm{~F}$ & 34 & Maxilla & 4 \\
\hline 2 & M & 63 & Right mandible & 3 \\
\hline 3 & $\mathrm{~F}$ & 32 & Right maxilla & 3 \\
\hline 4 & M & 37 & Left maxilla & 2 \\
\hline 5 & M & 43 & Left mandible & 4 \\
\hline 6 & M & 32 & Mandible & 7 \\
\hline 7 & $\mathrm{~F}$ & 44 & Left mandible & 7.5 \\
\hline 8 & M & 66 & Left maxilla & 3 \\
\hline 9 & M & 29 & Left maxilla & 2 \\
\hline 10 & M & 73 & Right maxilla & 5 \\
\hline 11 & $\mathrm{~F}$ & 51 & Right maxilla & 3 \\
\hline 12 & M & 58 & Right maxilla & 5 \\
\hline 13 & M & 47 & Left mandible & 3 \\
\hline 14 & M & 44 & Left mandible & 6 \\
\hline 15 & $\mathrm{~F}$ & 65 & Right mandible & 5 \\
\hline
\end{tabular}

M, male; F, female.

as follows: $2 \mu$ l anti-CD14-APC (cat. no. 555399; BD Pharmingen; BD Biosciences, Inc.), anti-CD4-APC-Cy7 (cat. no. 557871; BD Pharmingen; BD Biosciences, Inc.), anti-CD15-PE-Cy7 (cat. no. 560827; BD Pharmingen; BD Biosciences, Inc.) and $2 \mu \mathrm{l}$ Annexin V-PerCP-Cy5.5 (cat. no. 640936; BioLegend, Inc., San Diego, CA, USA). Additionally, Annexin V binding buffer containing calcium (BD Biosciences, Inc.) was used for Annexin V detection.
Table III. Summary of clinical features of patients with uninflamed odontogenic keratocysts.

\begin{tabular}{llclc}
\hline Patient no. & Sex & Age, years & \multicolumn{1}{c}{ Location } & $\begin{array}{c}\text { Cyst fluid } \\
\text { drained, ml }\end{array}$ \\
\hline 1 & F & 24 & Right mandible & 2 \\
2 & F & 33 & Maxilla & 3 \\
3 & F & 42 & Right mandible & 3 \\
\hline
\end{tabular}

M, male; F, female.

Table IV. Summary of clinical features of patients with focal inflamed odontogenic keratocysts.

\begin{tabular}{llllr}
\hline & & & & \\
Patient no. & Sex & Age, years & \multicolumn{1}{c}{ Location } & $\begin{array}{c}\text { Cyst fluid } \\
\text { drained, ml }\end{array}$ \\
\hline 1 & F & 24 & Left mandible & 2.5 \\
2 & F & 24 & Right mandible & 2 \\
3 & M & 32 & Left mandible & 4.5 \\
4 & M & 52 & Left mandible & 1 \\
5 & M & 79 & Right mandible & 5 \\
6 & M & 24 & Right mandible & 2 \\
7 & M & 11 & Right mandible & 5 \\
8 & F & 52 & Right maxilla & 2 \\
9 & F & 45 & Right mandible & 3 \\
10 & M & 34 & Left mandible & 0.8 \\
11 & F & 28 & Right mandible & 3 \\
12 & M & 37 & Left maxilla & 7 \\
13 & M & 26 & Right maxilla & 4 \\
14 & M & 24 & Left mandible & 1 \\
15 & F & 32 & Left mandible & 1 \\
16 & M & 16 & Right mandible & 2 \\
17 & F & 30 & Left maxilla & 2 \\
18 & M & 38 & Right mandible & 1 \\
19 & F & 27 & Maxilla & 1 \\
20 & M & 27 & Right maxilla & 1 \\
\hline
\end{tabular}

M, male; F, female.

These antibodies and binding buffers were mixed with samples for $30 \mathrm{~min}$ at $4^{\circ} \mathrm{C}$ in the dark. A total of $380 \mu \mathrm{l} \mathrm{PBS}$ was added to the CFMPs and then analyzed by flow cytometry. The results were calculated using FlowJo 9.3.2 software (FlowJo LLC, Ashland, Oregon, USA).

Cell culture and Jurkat cell-derived MP collection. Jurkat Clone E6-1 human T cells (an immortal lymphoma T-cell line) were grown in RPMI-1640 medium supplemented with $10 \%$ fetal bovine serum (FBS; Gibco; Thermo Fisher Scientific, Inc.) at $37^{\circ} \mathrm{C}$ in $5 \% \mathrm{CO}_{2}$. For the induction of apoptosis, $1 \mu \mathrm{M}$ staurosporine (Sigma-Aldrich; Merck KGaA, Darmstadt, Germany) or $1 \mu \mathrm{g} / \mathrm{ml}$ camptothecin (Sigma-Aldrich; 
Table V. Summary of clinical features of patients with odontogenic keratocysts for fibroblasts isolation.

\begin{tabular}{lcclc}
\hline Patient no. & Sex & Age, years & Location & Diameter, cm \\
\hline 1 & F & 35 & Left mandible & 2 \\
2 & M & 26 & Right maxilla & 3 \\
3 & F & 39 & Mandible & 4.5 \\
\hline
\end{tabular}

M, male; F, female.

Merck $\mathrm{KGaA}$ ) was added to the Jurkat cells. Following these treatments, cells were cultured for an additional $48 \mathrm{~h}$ without FBS culture medium and then the supernatant was centrifuged at $400 \mathrm{x}$ g for $5 \mathrm{~min}$ to remove cells (centrifuge 5810R; Eppendorf) at $4^{\circ} \mathrm{C}$. Supernatant was transferred to a fresh tube and recentrifuged again at $2,000 \mathrm{x}$ g for $20 \mathrm{~min}$ at $4^{\circ} \mathrm{C}$ (centrifuge 5810R; Eppendorf). The supernatant was centrifuged for $1 \mathrm{~h}$ at 50,000 x gat $4^{\circ} \mathrm{C}$ via the Avanti J-26 XP (Beckman Coulter, Inc.). The pellet was resuspended in PBS (Beyotime Institute of Biotechnology) and stored at $-80^{\circ} \mathrm{C}$.

Bicinchoninic acid assay (BCA) determination of Jurkat cell-derived MPs. Jurkat cell-derived MPs were lysed in radioimmunoprecipitation (RIPA) assay buffer (Thermo Fisher Scientific, Inc.) and exposed to sonication, and then the proteins were quantified using the BCA assay (cat. no., 23225; Pierce; Thermo Fisher Scientific, Inc.). In brief, $5 \mu 1$ albumin standard with concentrations of 2,000, 1,000, 500, 250, 125, 62.5, $0 \mathrm{ng} / \mu 1$ and $5 \mu 1$ Jurkat cell-derived MPs (concentrations to be determined) were added to the working solution and incubated for $30 \mathrm{~min}$ at $37^{\circ} \mathrm{C}$ in 96 -well plates. Next, the plate was read at $570 \mathrm{~nm}$ and the protein concentrations of Jurkat cell-derived MPs were calculated with the albumin standard proteins.

Cytokine antibody arrays. Jurkat cell-derived MPs and the Jurkat cell supernatants were analyzed on a Bio-Plex Pro Human Cytokine GrpI Panel 27-plex (\#M500KCAF0Y), according to the manufacturer's protocols (Bio-Plex MAGPIX System; Bio-Rad Laboratories, Inc., Hercules, CA, USA). Specifically, $50 \mu \mathrm{g}$ Jurkat cell-derived MPs and the Jurkat cell supernatants were loaded and tested. The cytokine concentrations were calculated using Bio-Plex Manager software 6.1 (Bio-Rad Laboratories, Inc.).

Fibroblast isolation and culture. Fibroblast cultures were established from fresh samples of OKCs from 3 patients and the procedure was approved by the review board of the Ethics Committee of Hospital of Stomatology, Wuhan University. The detailed information of the patients is listed in Table V. As previously described, but with modification (16), the OKC tissues were washed and cut into $5 \times 5-\mathrm{mm}^{2}$ cubes and plated into a T25 flask, which was incubated at $37^{\circ} \mathrm{C}$ for $2 \mathrm{~h}$. Next, 3 ml Dulbecco's modified Eagle's medium (DMEM; Gibco; Thermo Fisher Scientific, Inc.) containing $1 \%$ antibiotics $(100 \mathrm{U} / \mathrm{ml}$ penicillin and $100 \mu \mathrm{g} / \mathrm{ml}$ streptomycin;
Sigma-Aldrich; Merck KGaA) and 10\% FBS was gently added to the flask, and the medium was replaced every 3 days. The fibroblasts were observed at 5-7 days post-culture. Fibroblasts were isolated and routinely cultured in DMEM, containing $1 \%$ antibiotics and 10\% FBS. Fibroblasts from passages 4-6 were used for subsequent experiments.

Immunofluorescence. Fibroblasts were seeded onto glass slides (Nest, Beijing, China) and grown to $80 \%$ confluence in 12-well plates. Following 3 washes with PBS, the cells were fixed in $4 \%$ paraformaldehyde in PBS for $20 \mathrm{~min}$ at $37^{\circ} \mathrm{C}$, washed 3 times with PBS for $5 \mathrm{~min}$ and permeabilized with $0.2 \%$ Triton $\mathrm{X}-100$ for $10 \mathrm{~min}$ at room temperature. The cells were blocked with $0.5 \%$ bovine serum albumin for $1 \mathrm{~h}$ at $37^{\circ} \mathrm{C}$, prior to being incubated with monoclonal mouse antibodies against $\alpha$-smooth muscle actin ( $\alpha$-SMA; 1:100; cat. no. ZM-0003; OriGene Technologies, Inc., Beijing, China) and fibroblast activation protein (FAP; 1:100; cat. no. sc-65398; Santa Cruz Biotechnology, Inc., Dallas, TX, USA) overnight at $4^{\circ} \mathrm{C}$. The slides were washed again twice with PBS, prior to being incubated with fluorescence-labeled secondary antibodies (FITC-labeled goat anti-mouse IgG; 1:200; cat. no. A0568; Beyotime Institute of Biotechnology) in a dark chamber for $45 \mathrm{~min}$ at $37^{\circ} \mathrm{C}$. Cell nuclei were counterstained with 4',6-diamidino-2-phenylindole (DAPI) at room temperature for $15 \mathrm{~min}$. The glass coverslips were examined under a fluorescence microscope with appropriate filters.

Fibroblast treatments. OKC fibroblasts were plated and cultured in a T25 flask at a cell density of $2 \times 10^{5} / \mathrm{ml}$. When the OKC fibroblasts reached $50 \%$ confluence, they were co-cultured with staurosporine-treated Jurkat cell-derived MPs (5 or $10 \mu \mathrm{g} / \mathrm{ml})$ or with $100 \mathrm{ng} / \mathrm{ml}$ anti-human IL-15R $\alpha$ antibody (goat; cat. no. AF247; R\&D Systems, Inc., Minneapolis, MN, USA) for $72 \mathrm{~h}$ at $37^{\circ} \mathrm{C}$. For co-culture with Jurkat cells, OKC fibroblasts were plated and cultured in a T25 flask at a cell density of $2 \times 10^{5} / \mathrm{ml}$, together with the Jurkat cell suspensions at a cell density of $2 \times 10^{5} / \mathrm{ml}$ or $6 \times 10^{5} / \mathrm{ml}$, for a period of $72 \mathrm{~h}$ at $37^{\circ} \mathrm{C}$.

Cellular uptake assay. To investigate the biological functions of LMPs, cellular uptake assays were performed as described in our previous study (17). The OKC primary fibroblasts were labeled with CellMask (1:2,000; Thermo Fisher Scientific, Inc.) at $37^{\circ} \mathrm{C}$ for $30 \mathrm{~min}$ in the dark and cultured on cover slips in 12-well plates. LMPs $(5 \mu \mathrm{g})$ from Jurkat cells were labeled with CFSE at $37^{\circ} \mathrm{C}$ for $30 \mathrm{~min}$, prior to being added to the CellMask-labeled OKC fibroblasts, which were co-cultured for a period of $2 \mathrm{~h}$ at $37^{\circ} \mathrm{C}$. Subsequently, OKC fibroblasts were fixed in $4 \%$ paraformaldehyde for $10 \mathrm{~min}$ at room temperature, stained with DAPI for $15 \mathrm{~min}$ at $37^{\circ} \mathrm{C}$ and observed under a confocal microscope (Revolution XD; Andor, Belfast, UK) equipped with an Olympus IX 81 microscope (Olympus Corporation, Tokyo, Japan) and an EMCCD (iXon DU897U single photon detector; Andor).

Reverse transcription-quantitative polymerase chain reaction $(R T-q P C R)$. Isolation of total RNA, synthesis of cDNA and 
RT-qPCR were performed as previously described (18). Briefly, total RNA was extracted from OKC fibroblasts using TRIzol reagent (Invitrogen; Thermo Fisher Scientific, Inc.). Next, $2 \mu \mathrm{g}$ RNA was reverse transcribed to $20 \mu \mathrm{l}$ cDNA with random primer with RevertAid ${ }^{\mathrm{TM}}$ First Strand cDNA Synthesis kit (cat. no. K1622; Thermo Fisher Scientific, Inc.). Subsequently, one-fifth of the cDNA was used for PCR using FastStart Universal SYBR-Green Master mix (Roche Diagnostics, Basel, Switzerland) in a 7900HT Real-Time PCR system (Applied Biosystems; Thermo Fisher Scientific, Inc.). GAPDH was selected as an internal control. The primer nucleotide sequences for PCR were designed as follows: RANKL forward, 5'-TGATTCATGTAGGAGAATTAAACAGG-3' and reverse, 5'-GATGTGCTGTGATCCAACGA-3'; MMP-9 forward, 5'-TGTACCGCTATGGTTACACTCG-3' and reverse, 5'-GGC AGGGACAGTTGCTTCT-3'; and GAPDH forward, 5'-CGT CATGGGTGTGAACCATTGAGAAG-3' and reverse, 5'-GCA TGGACTGTGGTCATGAGTCCTT-3'. The thermal cycling conditions comprised $94^{\circ} \mathrm{C}$ for $30 \mathrm{sec}, 60^{\circ} \mathrm{C}$ for $1 \mathrm{~min}, 72^{\circ} \mathrm{C}$ for $1 \mathrm{~min}$ and a final elongation at $72^{\circ} \mathrm{C}$ for $10 \mathrm{~min}$, amplifying for 36 cycles. The $2^{-\Delta \Delta \mathrm{Cq}}$ method was used for the analysis of the data (19).

Enzyme-linked immunosorbent assay (ELISA). Human RANKL levels were determined in the supernatant of fibroblasts using a commercial ELISA kit (cat. no. CSB-E05125 h; Cusabio Technology LLC, Wuhan, China) following the manufacturer's protocols. Specifically, $5 \times 10^{5} \mathrm{OKC}$ fibroblasts were placed in 6-well plates with serum-free DMEM. For the experiments, fibroblasts were treated with LMPs (5 or $10 \mu \mathrm{g} / \mathrm{ml}$ ) for 24 or $48 \mathrm{~h}$. Next, $200 \mu \mathrm{l}$ of the supernatant of OKC fibroblasts were collected for ELISA assay.

Osteoclast formation assay. Raw264.7 cells obtained from the China Center for Type Culture Collection (Wuhan, China), were seeded onto slides at a density of 1,000 cells/well in 12-well plates and cultured with DMEM (Gibco; Thermo Fisher Scientific, Inc.) supplemented with $10 \%$ FBS containing $1 \%$ antibiotics $(100 \mathrm{U} / \mathrm{ml}$ penicillin and $100 \mu \mathrm{g} / \mathrm{ml}$ streptomycin; Sigma-Aldrich; Merck $\mathrm{KGaA}$ ) at $37^{\circ} \mathrm{C}$ with a supply of $5 \% \mathrm{CO}_{2}$ for 3 days. Next, the Raw264.7 cells were cultured with OKC fibroblast supernatant or Jurkat MP-treated fibroblasts supernatant $(10 \mu \mathrm{g} / \mathrm{ml} ; 48 \mathrm{~h})$ with or without anti-human sRANKL (1,000 ng/ml; R\&D Systems, Inc.). The medium was replaced every 2 days for 8 days. Cells were stained for tartrate-resistant acid phosphatase (TRAP; kit cat. no. 387A-1; Sigma-Aldrich; Merck KGaA), according to the manufacturer's protocol. TRAP-positive cells containing no less than three nuclei were counted as osteoclast-like cells. Raw264.7 cells routinely cultured in DMEM were used as a negative control.

Statistical analysis. Data were analyzed using GraphPad 6.0 software (GraphPad Software, Inc., La Jolla, CA, USA). Concentrations and proportions of LMPs, gene expression in fibroblasts, sRANKL concentrations and $\mathrm{TRAP}^{+}$multinucleated cells (MNCs) were compared using one-way analysis of variance, followed by Tukey's post hoc test. Data are presented as the mean \pm standard deviation. $\mathrm{P}<0.05$ was considered to indicate a statistically significant difference.

\section{Results}

Clinical characteristics of patients with cysts. A total of 12 patients with inflamed DCs (7 male and 5 female), 15 patients with RCs (10 male and 5 female), 3 patients with uninflamed OKCs (all female) and 20 patients with inflamed OKCs (12 male and 8 female) were enrolled in the present study (Tables I-IV). H\&E staining revealed inflammatory cell distributions among the inflamed DCs, RCs, and uninflamed and inflamed OKCs (Fig. 1A-D). The mean ages of the patients with inflamed DCs, RCs, and uninflamed and inflamed OKCs were $36 \pm 12,48 \pm 14,33 \pm 9$ and $33 \pm 15$ years, respectively. No significant differences in age or sex distribution were identified in the present study.

CFMP identification and analysis. CFMPs from patients with odontogenic cysts were purified by differential centrifugation. To directly visualize the CFMPs, TEM and CFSE fluorescence labeling were performed. CFMPs were membrane-bound vesicles with a round or ellipse structure by TEM (Fig. 1E). CFMPs could also be successfully stained by CFSE, indicating their envelope structure (Fig. 1F). Dynamic light scattering demonstrated that the purified CFMPs had a size distribution ranging between 100 and 1,000 nm (Fig. 1G). Using the Nile Red fluorescent particles with known diameters (0.7-0.9 $\mu \mathrm{m})$, the diameters of the CFMPs were determined, ranging between 100 and 1,000 nm (Fig. 1H).

Increased percentages of neutrophil-derived MPs in patients with inflamed $O K C$. For comparison of the subtypes of LMPs, three types of leukocyte cell (neutrophils, monocytes and lymphocytes) were analyzed by flow cytometry using anti-CD15, -CD14 and -CD4 staining combined with Annexin $\mathrm{V}$ staining (Fig. 2A-F). The results demonstrated that the percentages of $\mathrm{CD}^{+}$lymphocyte-derived CFMPs were significantly higher in patients with inflamed $\mathrm{OKCs}$ compared with those in patients with inflamed DCs (Fig. 2G). The percentages of monocyte-derived CFMPs showed no significant difference in patients with inflamed DCs, RCs, and inflamed or uninflamed OKCs (Fig. 2H). There was also an increase in the percentage of neutrophil-derived CFMPs in inflamed OKCs compared with that in RCs (Fig. 2I). Although the majority of the LMP concentrations of inflamed OKCs were higher, only the concentration of neutrophil-derived CFMPs was significantly higher than that of the inflamed DCs (Fig. 2J-L).

Increased release of MPs from Jurkat cells following staurosporine treatment. The release of MPs was first examined in Jurkat cells at the basal state and following treatments to induce apoptosis. A BCA assay demonstrated that $1 \times 10^{5}$ Jurkat cells released $2 \mathrm{ng} / \mu 1$ MPs constitutively. The concentration of MPs released from Jurkat cells was significantly increased up to $9 \mathrm{ng} / \mu \mathrm{l}$ following stimulation with staurosporine (Fig. 3A). Furthermore, TEM revealed a high density of bound membrane vesicles derived from staurosporine-treated Jurkat cells (Fig. 3B). However, there was no significant change in MPs concentration in the camptothecin-treated Jurkat cells compared with the basal state of the Jurkat cells. 

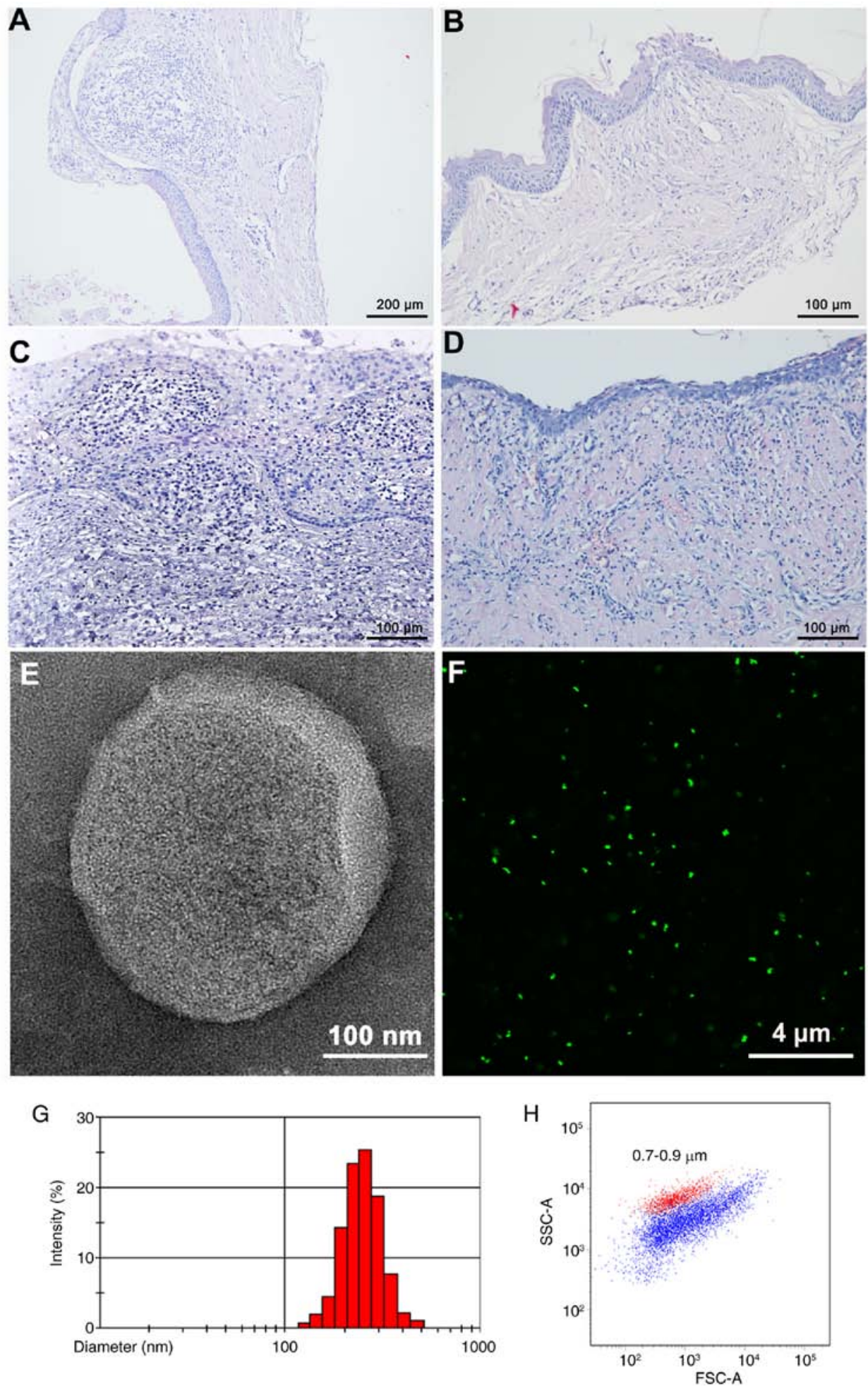

Figure 1. Isolation and identification of CFMPs from odontogenic lesions. Hematoxylin and eosin staining of (A) focal inflamed and (B) uninflamed odontogenic keratocysts, (C) radicular cysts and (D) inflamed dentigerous cysts. Characterization of CFMPs derived from patients with OKCs. (E) Transmission electronmicroscopy image of CFMPs purified from patients with OKC. (F) Fluorescence image demonstrating the successful labeling of CFMPs by the fluorescent dye carboxyfluorescein succinimidyl ester. (G) Dynamic light scattering was performed to assess the size distribution of CFMPs derived from patients with OKC. (H) The size distribution of CFMPs derived from patients with OKC was characterized by flow cytometry based on Nile Red fluorescent particles (red) with known diameters ranging between 0.7 and $0.9 \mu \mathrm{m}$. CFMP, cyst fluid microparticle; OKC, odontogenic keratocysts; SSC, side scatter; FSC, forward scatter.

Significant increases ininflammatory cytokines in Jurkat cell-derived MPs compared with that in Jurkat cell supernatants. To determine the difference between the Jurkat cell derived-MPs and the Jurkat cell supernatant, an equal weight of the Jurkat cell MPs and supernatants were determined by cytokine antibody arrays. The results demonstrated that certain cytokines, including IL-15, IL-8, interferon- $\gamma$ and macrophage inflammatory protein- $1 \beta$ were detected in Jurkat cell-derived MPs. Among them, the highest concentration was in IL-15 (Fig. 3C). 
A

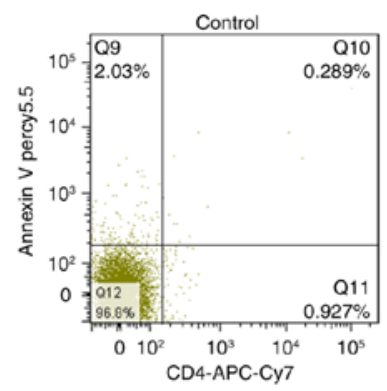

D

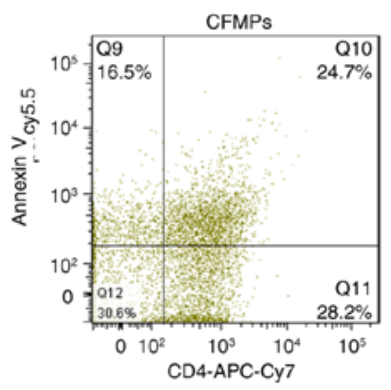

G

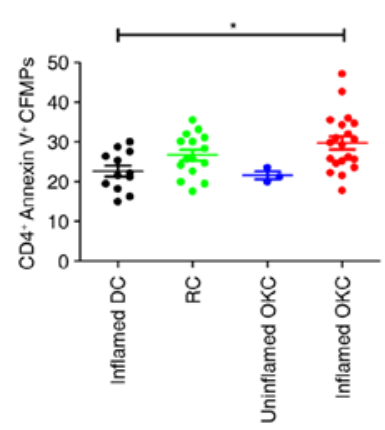

$J$

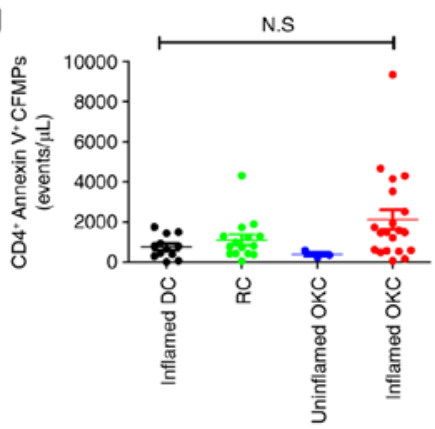

B

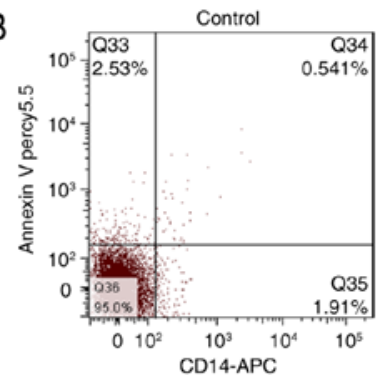

E

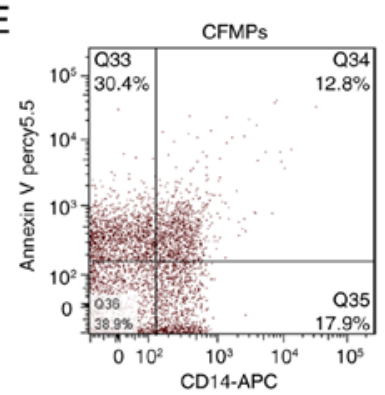

$\mathrm{H}$

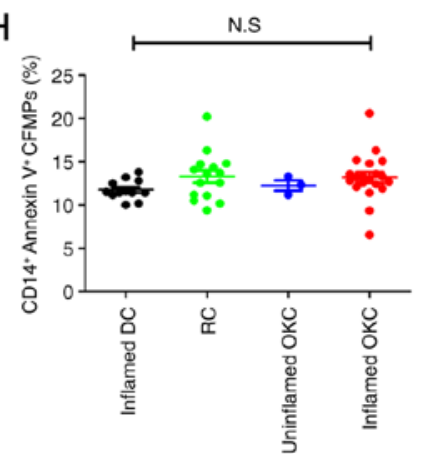

$\mathrm{K}$

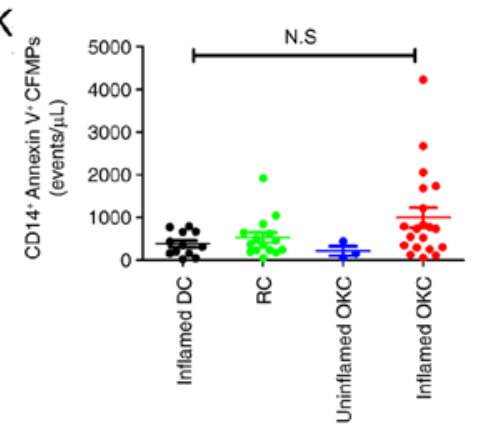

C

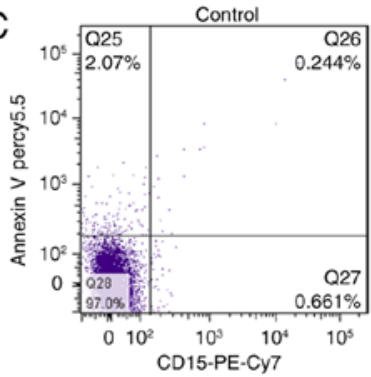

F

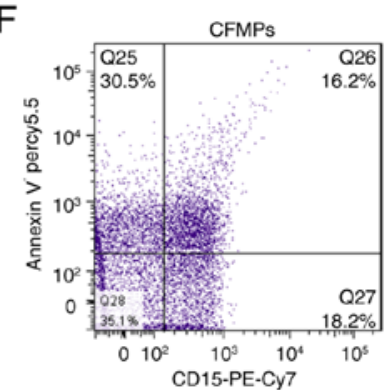

I

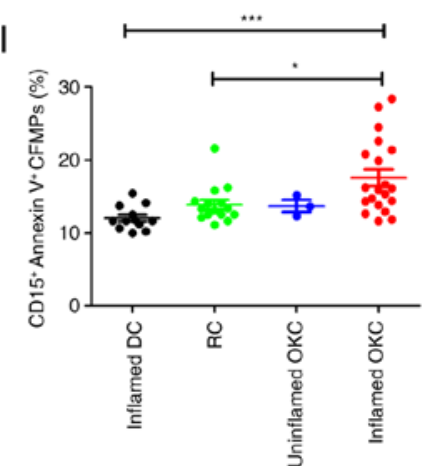

L

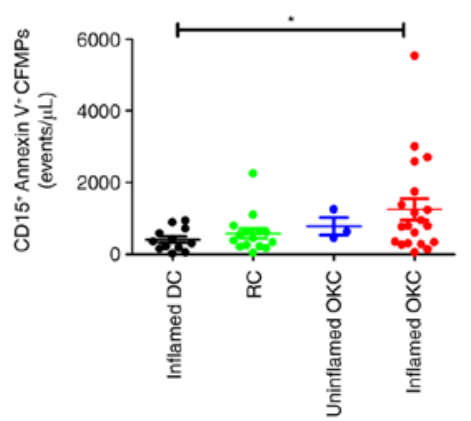

Figure 2. Quantification of the concentration and proportion of leukocyte-derived MPs within odontogenic lesions. Representative flow cytometric images for (A) unstained CD4+ lymphocyte-derived CFMPs, (B) unstained monocyte-derived CFMPs and (C) unstained neutrophil-derived CFMPs. Representative flow cytometric images for (D) stained $\mathrm{CD}^{+}$lymphocyte-derived CFMPs, (E) stained monocyte-derived CFMPs and (F) stained neutrophil-derived CFMPs. Quantitative analysis for the percentages of $(\mathrm{G}) \mathrm{CD}^{+} /$Annexin $\mathrm{V}^{+}$CFMPs, (H) CD14 $/$Annexin $\mathrm{V}^{+} \mathrm{CFMPs}$ and $(\mathrm{I}) \mathrm{CD}^{+} 5^{+} / \mathrm{Annexin} \mathrm{V}^{+} \mathrm{CFMPs}$ in uninflamed

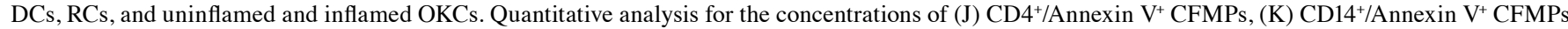
and (L) CD15 $/$ Annexin $\mathrm{V}^{+}$CFMPs in inflamed DCs, RCs, and uninflamed and inflamed OKCs. Data are presented as the mean \pm standard deviation. ${ }^{*} \mathrm{P}<0.05$ and ${ }^{* * * *} \mathrm{P}<0.001$. N.S, not significant; CFMPs, cyst fluid microparticles; OKCs, odontogenic keratocysts; DCs, dentigerous cysts; RCs, radicular cysts; $\mathrm{CD}$, cluster of differentiation, PE, phycoerythrin; APC, allophycocyanin; Cy7, cyanine 7.

Jurkat cell-derived MPs carrying IL-15 significantly promote MMP-9 and RANKL expression in OKC fibroblasts, and promote osteoclastogenesis. After 5-7 days of culturing from the cyst wall of OKCs, explants grew spindle-shaped fibroblast-like cells that were positive for $\alpha$-SMA and FAP staining, thereby confirming their mesenchymal origin (Fig. 3D and E). To determine the biological effects of Jurkat cell-derived MPs on primary OKC fibroblasts, a cellular uptake assay was performed. The results demonstrated that Jurkat cell-derived
MPs (green dots) were detected in the cytoplasm of OKC fibroblasts within $2 \mathrm{~h}$ (Fig. 3F). The bone resorption-related mRNAs were investigated, and the results indicated that the mRNA expression levels of MMP-9 and RANKL were increased in Jurkat cell-derived MPs-treated (5 or $10 \mu \mathrm{g} / \mathrm{ml}$ ) and Jurkat cell co-cultured $\mathrm{OKC}$ fibroblasts compared with that in the control group (Fig. 4A and B). Additionally, the mRNA levels of MMP-9 and RANKL were decreased by the use of anti-human IL-15R $\alpha$ antibody in the Jurkat cell-derived MP-treated group. 

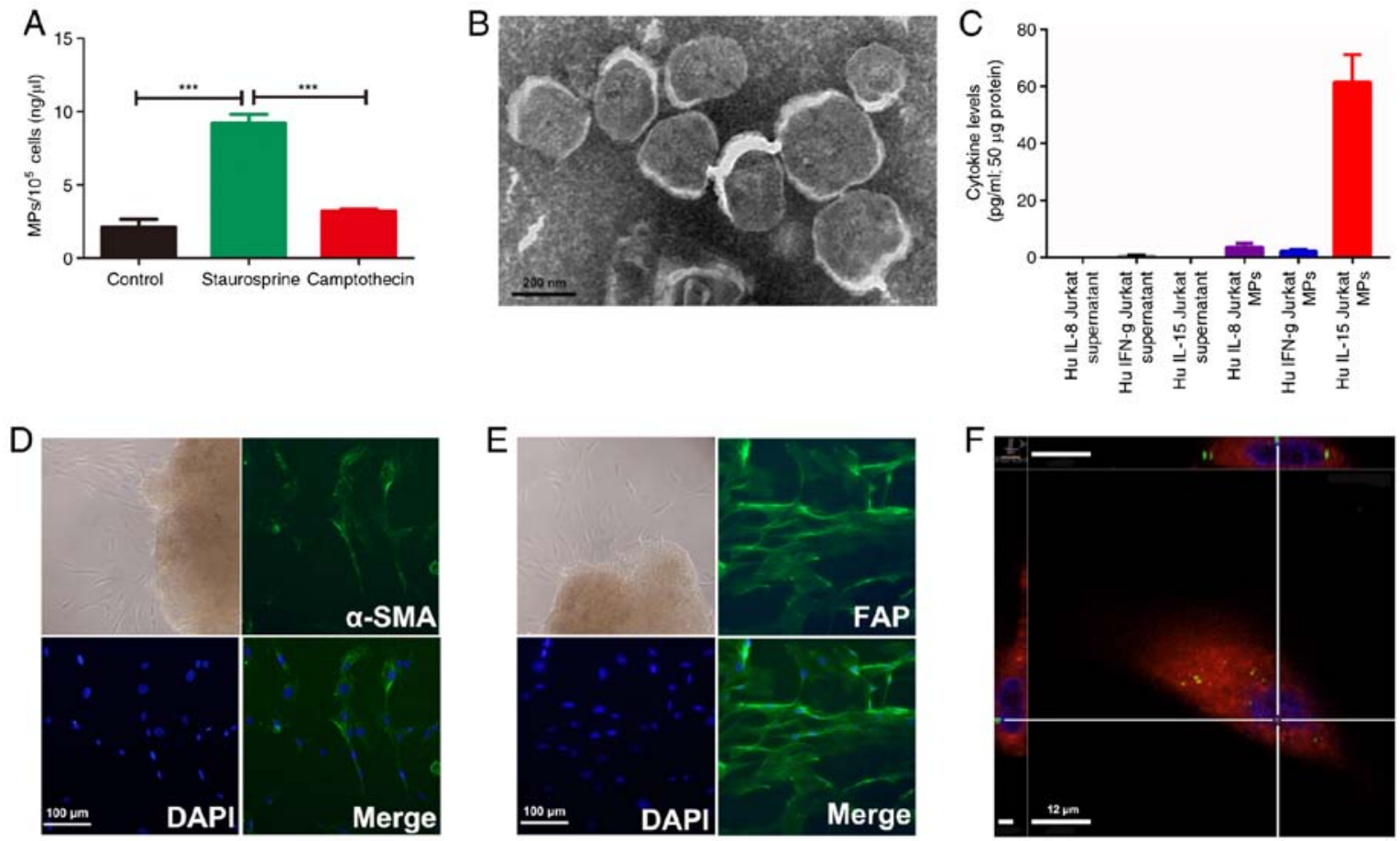

Figure 3. Apoptotic Jurkat MPs harbor a panel of cytokines that could be taken up by OKC fibroblasts. (A) Comparison of the Jurkat cell MPs concentrations in normal and staurosporine- and camptothecin-treated Jurkat cells. (B) Analysis of MPs from Jurkat cells by transmission electron microscopy revealed membrane-coated circular vesicles ranging in size between 200 and $400 \mathrm{~nm}$. (C) Cytokines levels were compared between Jurkat MPs and Jurkat supernatant. (D and E) Primary fibroblasts grew from explanted cyst wall tissue specimens after 5-7 days of culture; OKC fibroblasts were characterized by $\alpha$-SMA and FAP immunofluorescence staining. (F) Uptake assays for carboxy fluorescein succinimidyl ester-labeled CFMPs (green) were performed using OKC fibroblasts (red) as the recipient cells. Data are presented as the mean \pm standard deviation. ${ }^{* * *} \mathrm{P}<0.001$ vs. control group. CFMPs, cyst fluid microparticles; $\alpha$-SMA, $\alpha$-smooth muscle actin; FAP, fibroblast activation protein; OKC, odontogenic keratocysts; IL, interleukin; Hu, human.

Therefore, the Jurkat cell-derived MPs containing IL-15 could lead to higher levels of RANKL and MMP-9 mRNAs in OKC fibroblasts. Moreover, the Jurkat cell-derived MPs could significantly upregulate the RANKL levels within the supernatant of OKC fibroblasts. Specifically, the $10 \mu \mathrm{g} / \mathrm{ml}$ Jurkat MPs-treated OKC fibroblasts $(48 \mathrm{~h})$ showed approximately two-fold higher sRANKL within the supernatant of OKC fibroblasts than the that in the control group (Fig. 4C). In addition, Raw264.7 cells were co-cultured with normal OKC fibroblast supernatant and Jurkat MPs-treated fibroblast supernatant $(10 \mu \mathrm{g} / \mathrm{ml}$, $48 \mathrm{~h}$ ). TRAP ${ }^{+}$MNCs were detected in all co-culture groups. Specifically, the supernatant of the Jurkat cell MP-treated fibroblast group had more abundant $\mathrm{TRAP}^{+} \mathrm{MNCs}(14 \pm 5$ cells) than the OKC fibroblast supernatant group ( $7 \pm 1$ cells) for each well (Fig. 4D). The addition of anti-sRANKL to Jurkat cell MPs-treated fibroblast supernatant decreased the trend of TRAP ${ }^{+}$MNCs (3 \pm 3 ) (Fig. 4D).

\section{Discussion}

MPs are released by cell membrane budding, which could occur either spontaneously or in response to various stimuli (9). Various body fluids, including saliva, blood, pleural fluid, urine, synovial fluid and ascites contain MPs (20). In the present study, the distribution of MPs shed from neutrophils, monocytes and lymphocytes in human cyst fluids was analyzed. The study demonstrated an accumulation of LMPs in patients with inflamed OKC. In addition, Jurkat cell-derived MPs carrying IL-15 and other cytokines could stimulate the expression of
RANKL and MMP-9 in OKC fibroblasts, which accelerates osteoclastogenesis (Fig. 4E).

LMPs are generated from the neutrophils, monocytes/ macrophages and lymphocytes, which are associated with the development of various diseases. It has previously been confirmed that LMPs could stimulate the expression of proinflammatory genes in endothelial cells, leading to the production of cytokines in vitro (21). In inflammatory diseases, including Ebola fever and sepsis, the number of tissue factor-positive monocyte-derived MPs $\left(\mathrm{CD} 14^{+}\right)$is increased and this is associated with disease progression $(22,23)$. Additionally, there have been a few studies on the effect of LMPs on fibroblasts. In rheumatoid arthritis, MPs from the Jurkat cells and U937 monocytes could significantly induce MMP-9 mRNA expression and the expression of cytokines, including IL-6, IL-8 and MCP, which are released by synovial fibroblasts (24). Also, the presence of sonic hedgehog (Shh) in MPs generated from activated/apoptotic human T lymphocytes (CEM T-cell line) had been reported $(25,26)$. However, the present study employed the Jurkat cell line, and without phytohemagglutinin and phorbol-12-myristate-13-acetate activation treatment, the results showed no expressions of Shh in Jurkat cell-derived MPs by western blot assay.

During the release of MPs, the asymmetric distribution of phospholipids of the plasma membrane is lost, leading to PS exposure (27). Typically, MPs expose the anionic phospholipid PS on their membrane surface, enabling their detection by Annexin V staining (28). The cyst fluid of OKCs usually presents as a semi-solid form, and is characterized by a large 
A

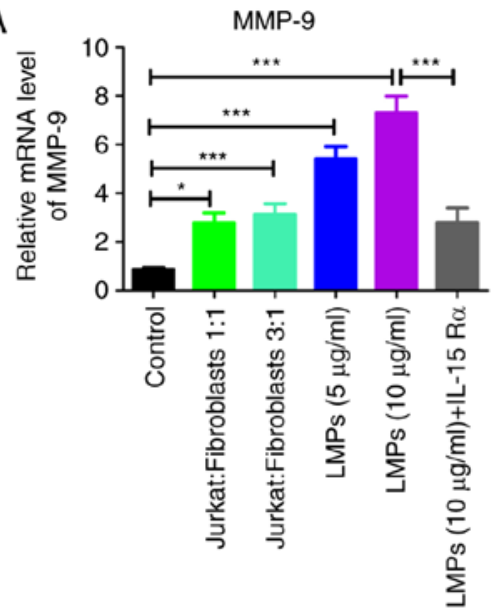

B

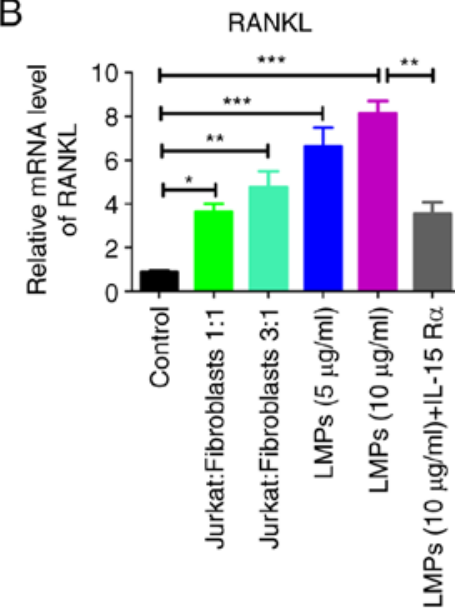

C

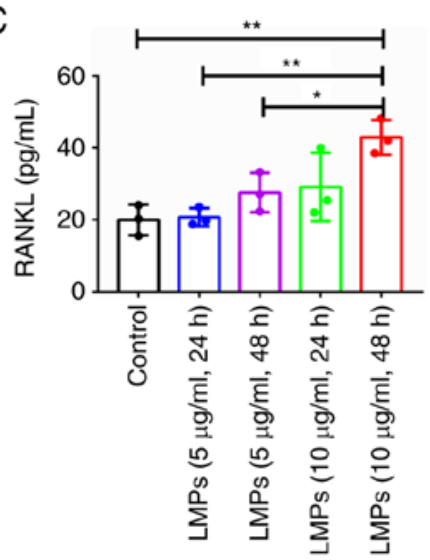

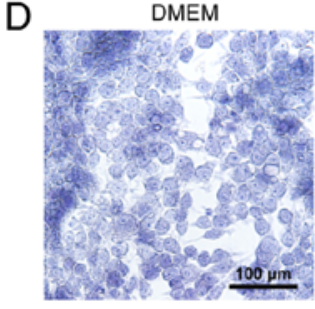

LMPs treated fibroblasts supernatant

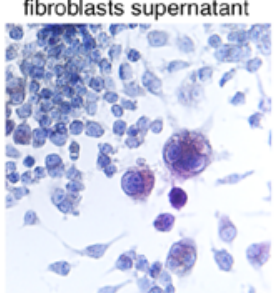

Normal fibroblasts supernatant

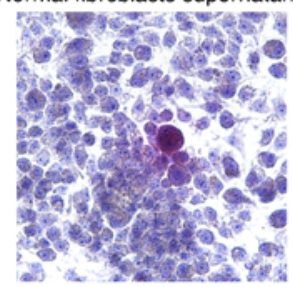
supernatant+anti-sRANKL

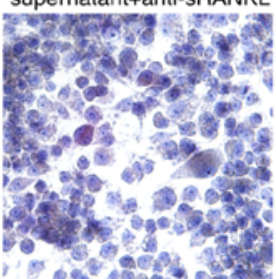

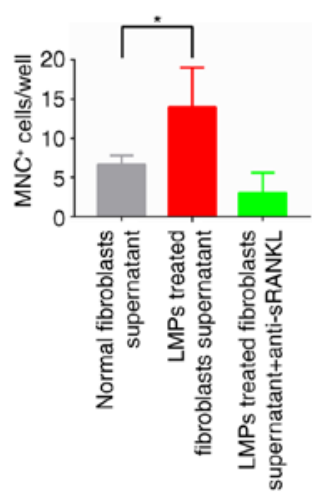

E

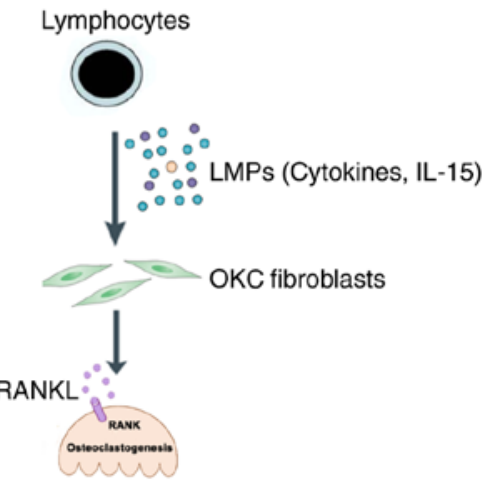

Figure 4. Apoptotic Jurkat MPs could boost RANKL expression of OKC fibroblasts and stimulate osteoclastogenesis. The OKC fibroblasts were treated with Jurkat cell-derived MPs and Jurkat cell suspension for $72 \mathrm{~h}$, and the (A) MMP-9 and (B) RANKL mRNAs were analyzed by reverse transcription-quantitative polymerase chain reaction. The results are presented as the relative ratio to the control group. Data are presented as the mean \pm standard deviation. ${ }^{*} \mathrm{P}<0.05$, ${ }^{* *} \mathrm{P}<0.01$ and ${ }^{* * *} \mathrm{P}<0.001$ vs. control group. (C) The level of sRANKL was determined in the supernatant from the Jurkat MP-treated fibroblasts by ELISA assay. (D) The $\mathrm{MNC}^{+}$cells were determined by TRAP staining in fibroblasts supernatant and Raw264.7 cell co-culture systems. (E) Lymphocyte-derived MPs as ostoclastogenesis players in the pathogenesis of odontogenic keratocysts. MPs are released from infiltrating leucocytes upon activation or apoptosis, and accumulate in the cyst fluid of inflamed OKCs. LMPs carry IL-15 and other cytokines activate fibroblasts of OKC, and potently stimulate MMP-9 and RANKL expression. Aberrant activation of fibroblasts by LMPs may therefore directly contribute toward bone destruction in OKC. sRANKL, soluble receptor activator of nuclear factor-кB ligand; MMP-9, matrix metallopeptidase 9; LMP, leukocyte-derived microparticle; DMEM, Dulbecco's modified Eagle's medium; MNC, multinucleated cell; TRAP, tartrate-resistant acid phosphatase; OKC, odontogenic keratocyst.

number of keratins (29). Due to the similar size distributions, it is difficult to distinguish MPs from protein fragments within the body fluids, which otherwise results in a significant amount of background noise for the quantification of MPs (30). Therefore, the present study defined CFMPs using positive staining for Annexin $\mathrm{V}$ in order to distinguish MPs from cell debris or precipitates, as previously described (30). In addition, as MPs carry surface membrane antigens from their donor cells, the present study tested the various cellular origins of MPs using Annexin V and specific markers, including CD15 (neutrophil marker), CD14 (monocyte marker) and CD4 (lymphocyte marker).

It has been demonstrated that LMPs serve a role in the pathogenesis of bone-related diseases (24). Previous studies have confirmed that activated $\mathrm{T}$ cells could activate osteoclast precursors to facilitate bone resorption $(31,32)$. Therefore, it is likely that the enhanced activation of leucocytes observed in patients with odontogenic cysts results in an increased release of MPs from these cells. In agreement with this hypothesis, the present study detected higher proportions of LMPs in inflamed OKCs. However, it is unclear why the number of LMPs in RCs was not as high as that in the focal inflamed OKCs, since diffused inflammatory cell infiltration has always been detected in RCs. Theoretically, more inflammatory cells are present in the tissue of RCs, which are located more diffusely than focal inflamed OKCs. It may be that the different microenvironments in the RCs and OKCs influence the release of LMPs. Additionally, inflamed OKCs exhibited a higher percentage and concentration of LMPs than uninflamed OKCs. However, due to the small group of patients with uninflamed OKC, this difference was not statistically significant.

To mimic the leukocyte-derived MPs, the present study collected the MPs in untreated and staurosporine- and camptothecin-treated Jurkat cells, an immortalized human 
T lymphocyte cell line. In line with the results of a previous study (33), the staurosporine-treated Jurkat cells exhibited a significantly higher concentration of MPs than the normal and camptothecin-treated groups. However, the contents of the Jurkat MPs, particularly the cytokine profiles of Jurkat cell-derived MPs, remain unknown. By using cytokine antibody arrays, the present study demonstrated that a panel of inflammatory cytokines was significantly upregulated compared with the Jurkat cell supernatant. Among them, IL-15, with the highest concentration, was detected in the Jurkat MPs. IL-15 has chemoattractant and pro-inflammatory properties, and may promote bone destruction (34). In line with this view, IL-15 was previously shown to stimulate the formation of mature osteoclasts in rat bone marrow cultures, and blocking IL-15 relieved the destruction of cartilage and bone $(35,36)$. IL-15 could also induce the RANKL expression of synovial fibroblasts, which ultimately facilitates the process of osteoclastogenesis (37). In the present study, the Jurkat cell-derived MPs-treated fibroblasts exhibited elevated expression of MMP-9 and RANKL mRNAs compared with the Jurkat cell co-cultured OKC fibroblasts, indicating that Jurkat-derived MPs are important message carriers in the crosstalk between OKC fibroblasts and T lymphocyte cells. Following the IL-15 blocking assay, the mRNA levels of MMP-9 and RANKL were significantly decreased, indicating that the Jurkat cell-derived MPs could carry IL-15 and induce the expression of bone resorption-related mRNAs. Also, significant elevation of sRANKL was detected in the Jurkat MPs-treated OKC fibroblasts. In addition, the co-culture experiment revealed that Jurkat MPs-treated fibroblasts had greater potential to promote osteoclastogenesis via RANKL. Therefore, MPs serve as novel communication tools that contribute toward the pathogenesis of OKCs by inducing RANKL and MMP-9 expression of OKC fibroblasts, which promote bone resorption. The present study reveals a novel mechanism between the inflammation and bone resorption of OKCs.

\section{Acknowledgements}

The authors would like to thank technician Juan Min from Wuhan Institute of Virology (Wuhan, China) for supporting the flow cytometric analysis.

\section{Funding}

This research was supported by the National Natural Science Foundation of China (grant no. 81570994).

\section{Availability of data and materials}

The datasets used during the present study are available from the corresponding author upon reasonable request.

\section{Authors' contributions}

QWM and LZZ were responsible for the conception and design of the study, manuscript preparation and submission. YZ, YYZ, JYL, YFZ and BL were responsible for the data interpretation. YFZ and BL revised the manuscript and all authors agreed to submit for peer review.

\section{Ethics approval and consent to participate}

The use of human samples was approved by the Review Board of the Medical Ethics Committee of the Hospital of Stomatology, Wuhan University (Wuhan, China). All patients agreed to participate in the study and signed informed consent forms.

\section{Patient consent for publication}

Not applicable.

\section{Competing interests}

The authors declare that they have no competing interests.

\section{References}

1. Shear M: The aggressive nature of the odontogenic keratocyst: Is it a benign cystic neoplasm? Part 1 . Clinical and early experimental evidence of aggressive behaviour. Oral Oncol 38: 219-226, 2002.

2. Mendes R, Carvalho J and van der Waal I: Characterization and management of the keratocystic odontogenic tumor in relation to its histopathological and biological features. Oral Oncol 46: 219-225, 2010

3. de Oliveira MG, Lauxen Ida S, Chaves AC, Rados PV and Sant'Ana Filho M: Odontogenic epithelium: immunolabeling of $\mathrm{Ki}-67$, EGFR and survivin in pericoronal follicles, dentigerous cysts and keratocystic odontogenic tumors. Head Neck Pathol 5: $1-7,2011$

4. Kramer IR, Pindborg JJ and Shear M: The WHO histological typing of odontogenic tumours. A commentary on the second edition. Cancer 70: 2988-2994, 1992.

5. Scalas D, Roana J, Boffano P, Mandras N, Gallesio C, Amasio M, Banche G, Allizond V and Cuffini AM: Bacteriological findings in radicular cyst and keratocystic odontogenic tumour fluids from asymptomatic patients. Arch Oral Biol 58: 1578-1583, 2013.

6. Avelar RL, Antunes AA, Carvalho RW, Bezerra PG, Oliveira Neto PJ and Andrade ES: Odontogenic cysts: A clinicopathological study of 507 cases. J Oral Sci 51: 581-586, 2009.

7. Jurisic V, Terzic T, Colic S and Jurisic M: The concentration of TNF-alpha correlate with number of inflammatory cells and degree of vascularization in radicular cysts. Oral Dis 14: 600-605, 2008.

8. Martins CA, Rivero ER, Dufloth RM, Figueiredo CP and Vieira DS: Immunohistochemical detection of factors related to cellular proliferation and apoptosis in radicular and dentigerous cysts. J Endod 37: 36-39, 2011.

9. Colombo M, Raposo G and Théry C: Biogenesis, secretion, and intercellular interactions of exosomes and other extracellular vesicles. Annu Rev Cell Dev Biol 30: 255-289, 2014.

10. Angelillo-Scherrer A: Leukocyte-derived microparticles in vascular homeostasis. Circ Res 110: 356-369, 2012.

11. Ardoin SP, Shanahan JC and Pisetsky DS: The role of microparticles in inflammation and thrombosis. Scand J Immunol 66: 159-165, 2007.

12. Iatrou IA, Legakis N, Ioannidou E and Patrikiou A: Anaerobic bacteria in jaw cysts. Br J Oral Maxillofac Surg 26: 62-69, 1988.

13. Wright JM and Vered M: Update from the 4th edition of the world health organization classification of head and neck tumours: Odontogenic and maxillofacial bone tumors. Head Neck Pathol 11: 68-77, 2017.

14. Ren JG, Man QW, Zhang W, Li C, Xiong XP, Zhu JY, Wang WM, Sun ZJ, Jia J, Zhang WF, et al: Elevated level of circulating platelet-derived microparticles in oral cancer. J Dent Res 95: 87-93, 2016.

15. Chen G, Zhu JY, Zhang ZL, Zhang W, Ren JG, Wu M, Hong ZY, Lv C, Pang DW and Zhao YF: Transformation of cell-derived microparticles into quantum-dot-labeled nanovectors for antitumor siRNA delivery. Angew Chem Int Ed Engl 54: 1036-1040, 2015. 
16. Wang $\mathrm{HC}$ and $\mathrm{Li} \mathrm{TJ}$ : The growth and osteoclastogenic effects of fibroblasts isolated from keratocystic odontogenic tumor. Oral Dis 19: 162-168, 2013.

17. Zhang W, Yu ZL, Wu M, Ren JG, Xia HF, Sa GL, Zhu JY, Pang DW, Zhao YF and Chen G: Magnetic and folate functionalization enables rapid isolation and enhanced tumor-targeting of cell-derived microvesicles. ACS Nano 11: 277-290, 2017.

18. Zhu JY, Ren JG, Zhang W, Wang FQ, Cai Y, Zhao JH, Chen G and Zhao YF: Characterization of microparticles in patients with venous malformations of the head and neck. Oral Dis 23: 110-119, 2017.

19. Livak KJ and Schmittgen TD: Analysis of relative gene expressiondata using real-time quantitative PCR and the $2^{-{ }^{-\Delta} \Delta C_{\mathrm{T}}}$ method. Methods 25: 402-408, 2001.

20. Gong J, Jaiswal R, Dalla P, Luk F and Bebawy M: Microparticles in cancer: A review of recent developments and the potential for clinical application. Semin Cell Dev Biol 40: 35-40, 2015.

21. Mesri M and Altieri DC: Leukocyte microparticles stimulate endothelial cell cytokine release and tissue factor induction in a JNK1 signaling pathway. J Biol Chem 274: 23111-23118, 1999.

22. Geisbert TW, Young HA, Jahrling PB, Davis KJ, Kagan E and Hensley LE: Mechanisms underlying coagulation abnormalities in ebola hemorrhagic fever: Overexpression of tissue factor in primate monocytes/macrophages is a key event. J Infect Dis 188: $1618-1629,2003$.

23. Nieuwland R, Berckmans RJ, McGregor S, Böing AN, Romijn FP, Westendorp RG, Hack CE and Sturk A: Cellular origin and procoagulant properties of microparticles in meningococcal sepsis. Blood 95: 930-935, 2000.

24. Distler JH, Jüngel A, Huber LC, Seemayer CA, Reich CF III, Gay RE, Michel BA, Fontana A, Gay S, Pisetsky DS, et al: The induction of matrix metalloproteinase and cytokine expression in synovial fibroblasts stimulated with immune cell microparticles. Proc Natl Acad Sci USA 102: 2892-2897, 2005.

25. Agouni A, Mostefai HA, Porro C, Carusio N, Favre J, Richard V, Henrion D, Martinez MC and Andriantsitohaina R: Sonic hedgehog carried by microparticles corrects endothelial injury through nitric oxide release. FASEB J 21: 2735-2741, 2007.

26. Martinez MC, Larbret F, Zobairi F, Coulombe J, Debili N, Vainchenker W, Ruat M and Freyssinet JM: Transfer of differentiation signal by membrane microvesicles harboring hedgehog morphogens. Blood 108: 3012-3020, 2006.

27. Piccin A, Murphy WG and Smith OP: Circulating microparticles: Pathophysiology and clinical implications. Blood Rev 21: 157-171, 2007.

28. Mallat Z, Hugel B, Ohan J, Leseche G, Freyssinet JM and Tedgui A: Shed membrane microparticles with procoagulant potential in human atherosclerotic plaques: A role for apoptosis in plaque thrombogenicity. Circulation 99: 348-353, 1999.
29. Aragaki T, Michi Y, Katsube K, Uzawa N, Okada N, Akashi T, Amagasa T, Yamaguchi A and Sakamoto K: Comprehensive keratin profiling reveals different histopathogenesis of keratocystic odontogenic tumor and orthokeratinized odontogenic cyst. Hum Pathol 41: 1718-1725, 2010.

30. Dey-Hazra E, Hertel B, Kirsch T, Woywodt A, Lovric S, Haller H, Haubitz M and Erdbruegger U: Detection of circulating microparticles by flow cytometry: Influence of centrifugation, filtration of buffer, and freezing. Vasc Health Risk Manag 6: 1125-1133, 2010.

31. Park JC, Kim BK, Jung IH, Choi E and Kim CS: Alveolar bone resorption induced by $\mathrm{CD} 4{ }^{+} \mathrm{CD} 45 \mathrm{RB}$ high-density T-cell transfer in immunocompromised mice. J Periodontol 85: e339-e347, 2014.

32. Wisitrasameewong W, Kajiya M, Movila A, Rittling S, Ishii T, Suzuki M, Matsuda S, Mazda Y, Torruella MR, Azuma MM, et al: DC-STAMP is an osteoclast fusogen engaged in periodontal bone resorption. J Dent Res 96: 685-693, 2017.

33. Reich CF III and Pisetsky DS: The content of DNA and RNA in microparticles released by Jurkat and HL-60 cells undergoing in vitro apoptosis. Exp Cell Res 315: 760-768, 2009.

34. Okabe I, Kikuchi T, Mogi M, Takeda H, Aino M, Kamiya Y, Fujimura T, Goto H, Okada K, Hasegawa Y, et al: IL-15 and RANKL play a synergistically important role in osteoclastogenesis. J Cell Biochem 118: 739-747, 2017.

35. Ferrari-Lacraz S, Zanelli E, Neuberg M, Donskoy E, Kim YS, Zheng XX, Hancock WW, Maslinski W, Li XC, Strom TB, et al: Targeting IL-15 receptor-bearing cells with an antagonist mutant IL-15/Fc protein prevents disease development and progression in murine collagen-induced arthritis. J Immunol 173: 5818-5826, 2004.

36. Ogata Y, Kukita A, Kukita T, Komine M, Miyahara A, Miyazaki S and Kohashi O: A novel role of IL-15 in the development of osteoclasts: Inability to replace its activity with IL-2. J Immunol 162: 2754-2760, 1999.

37. Park MK, Her YM, Cho ML, Oh HJ, Park EM, Kwok SK, Ju JH, Park KS, Min DS, Kim HY, et al: IL-15 promotes osteoclastogenesis via the PLD pathway in rheumatoid arthritis. Immunol Lett 139: 42-51, 2011.

This work is licensed under a Creative Commons Attribution-NonCommercial-NoDerivatives 4.0 International (CC BY-NC-ND 4.0) License. 